\title{
Comparison of Ventilation and Voice Outcomes between Unilateral Laryngeal Pacing and Unilateral Cordotomy for the Treatment of Bilateral Vocal Fold Paralysis
}

\author{
Yike Li ${ }^{a} \quad$ Elizabeth C. Pearce ${ }^{a}$ Rajshri Mainthia a Sanjay M. Athavale ${ }^{a}$ \\ Jennifer Dang ${ }^{a} \quad$ Daniel H. Ashmead ${ }^{b} \quad$ C. Gaelyn Garrett ${ }^{a}$ \\ Bernard Rousseau $^{a} \quad$ Cheryl R. Billante ${ }^{a}$ David L. Zealear ${ }^{a}$ \\ Departments of a Otolaryngology and ${ }^{b}$ Hearing and Speech Sciences, Vanderbilt University, \\ Nashville, Tenn., USA
}

\section{Key Words}

Ventilation · Voice outcome $\cdot$ Unilateral laryngeal pacing $\cdot$ Unilateral cordotomy

\begin{abstract}
Background/Aims: Rehabilitation of the bilaterally paralyzed human larynx remains a complex clinical problem. Conventional treatment generally involves surgical enlargement of the compromised airway, but often with resultant dysphonia and risk of aspiration. In this retrospective study, we compared one such treatment, posterior cordotomy, with unilateral laryngeal pacing: reanimation of vocal fold opening by functional electrical stimulation of the posterior cricoarytenoid muscle. Methods: Postoperative peak inspiratory flow (PIF) values and overall voice grade ratings were compared between the two surgical groups, and pre- and postoperative PIF were compared within the pacing group. Results: There were 5 patients in the unilateral pacing group and 12 patients in the unilateral cordotomy group. Within the pacing group, postoperative PIF values were significantly improved from preoperative PIF values $(p=0.04)$ without a significant effect on voice (grade; $p=0.62$ ). Within the pacing group, the mean postoperative PIF value was significantly higher than that in the cordotomy group $(p=$ 0.05 ). Also, the mean postoperative overall voice grade values in the pacing group were significantly lower (better) than those of the cordotomy group $(p=0.03)$. Conclusion: Unilateral pacing appears to be an effective treatment superior to posterior cordotomy with respect to postoperative ventilation and voice outcome measures.




\section{Introduction}

Despite recent advances in otolaryngology, rehabilitation of the paralyzed larynx remains a complex clinical problem. Unilateral vocal fold paralysis is more common than bilateral vocal fold paralysis (BVFP), yet the exact incidence of BVFP is unknown in the current literature. Etiologies of vocal fold paralysis include iatrogenic injury during thyroid, neck, and thoracic surgery, intubation, trauma, neurologic disorders, and extralaryngeal malignancies [1]. While normal ventilation and voice can be relatively spared with only one vocal fold paralyzed, reduction of airway area in BVFP can be life-threatening.

The recurrent laryngeal nerve carries motor fibers that innervate the abductor [posterior cricoarytenoid (PCA)] muscle of the vocal folds. Damage to bilateral nerves compromises this 'opening' function and arrests the vocal folds in a near-closed position. Both voice and airway are affected, and airway compromise is often severe enough to warrant tracheotomy to relieve inspiratory stridor and dyspnea [2-4]. If immobility is permanent and the patient suffers from a large degree of airway compromise, a tracheotomy may be necessary for the patient to avoid living with a tenuous and limited airway. Although permanent tracheostomy provides the best possible airway from a size standpoint, complications include tracheal stenosis, chronic infection, and psychosocial impairment [5-7]. For this reason, laryngeal surgery is offered to enlarge the airway and improve breathing through the mouth or nose.

Surgical treatment options for BVFP include temporizing, reversible treatments and/or permanent treatments. Reversible treatment options include the previously mentioned tracheostomy, endo-extralaryngeal suture lateralization as popularized by Lichtenberger and Toohill [8], or laryngeal botox injection in carefully selected cases [9]. Long-term, permanent surgical solutions include endoscopic laser arytenoidectomy [10], endoscopic partial arytenoidectomy [11], arytenoid abduction [12,13], posterior cordotomy [14], and laryngeal selective reinnervation [15-18]. As well, selective reinnervation for BVFP has been described in animal $[16,19]$ and cadaver models [20]. While human studies are sparse due to the variability and complexity of the nerve supply and the procedure [17], Marie [18] does describe successful selective reinnervation in 12 human patients with BVFP and noted improvement in patient dyspnea.

Endoscopic arytenoidectomy, and more commonly posterior cordotomy, are currently regarded as the standard of care for surgically enlarging the airway in the setting of BVFP. The posterior cordotomy procedure was the preferred surgical treatment for BVFP by $91 \%$ of fellowship-trained laryngologists in a recent survey [21], and at our institution, it was the most commonly performed treatment for BVFP during the study period. However, both arytenoidectomy and cordotomy have inherent limitations [22-25]: vibratory characteristics of the operated vocal fold are permanently altered, and thus patients often complain of a weak, breathy, and easily fatigued voice [22]. Scarring and granulation tissue can occur at the site, especially in repeat procedures, further worsening voice quality and potentially renarrowing the surgically improved airway [14]. Such limitations have prompted investigation into the previously mentioned novel techniques, and what we believe provides a more physiologic, dynamic approach to laryngeal rehabilitation: reanimation of the paralyzed vocal fold by functional electrical stimulation of the PCA muscle.

In 1996, our laboratory performed the first successful human implantation of a laryngeal pacing device that involved direct electrical stimulation of one of the PCA muscles in patients with BVFP. This approach has been termed 'unilateral pacing' [26]. The electrodes were connected to a neurostimulator device that provided stimulation of the PCA during the inspiratory phase of respiration to abduct the vocal fold. During noninspiratory phases, stimulation ceased and the vocal fold passively relaxed to the midline to allow for normal voice production and airway protection. Subsequent studies culminated in results from 6 human 
subjects that demonstrated the safety and efficacy of laryngeal pacing, with restoration of a functional airway through the mouth or nose.

The aim of this study was to retrospectively compare measurements of pulmonary function and voice quality between patients with bilateral vocal fold paralysis treated with unilateral vocal fold pacing and the current approach of cordotomy.

\section{Methods}

A retrospective chart review was carried out after approval from the Institutional Review Board at Vanderbilt University Medical Center. Records were obtained from 5 unilateral pacing patients, implanted between 1995 and 1997. Specific data extracted from the charts included pulmonary function tests with peak inspiratory flow rate (PIF), and overall voice grade ratings of voice quality obtained from electronic recordings of patients reading the Rainbow Passage. The records of cordotomy patients treated between 2009 and 2010 were reviewed, and the same pulmonary function data and overall voice grade ratings of audioclips of each patient reading the Rainbow Passage [27] were analyzed. Due to the retrospective design of this study, the time from surgical intervention to ventilation and voice testing varied across patients.

\section{Pulmonary Function Tests}

Pulmonary function assessment was performed by trained technicians in the Department of Pulmonary Medicine at Vanderbilt University (Sensor Medics, model 2200). Both pre- and postoperative results were collected for pacing patients, and postoperative results for cordotomy patients. The volume and rate of air exchange were measured during a maximum forced expiration followed by a maximum forced inspiration. PIF was measured through the mouth with the patient's nose and tracheostomy site (if any) occluded. For pacing patients, ventilatory measures were made with the neurostimulator device on. The PIF value taken from the inspiratory phase of the flow-volume loop was considered the critical indicator of glottal resistance to inspiratory flow [26]. The spirometry software automatically calculated 'best PIF' after each patient had performed 3-4 volume loops, and this value was used for each calculation.

\section{Voice Assessment}

All available previously recorded digital audiotaped voice samples of each patient reading the standardized Rainbow Passage were collected from the charts of both pacing and cordotomy patients. These were de-identified and randomly ordered into an audio-only compilation of all patients' audioclips, with 3 clips entered twice to establish interrater reliability. The compiled audioclips were presented to 3 trained speech-language pathologists who served as perceptual judges and were blinded to treatment group assignment. Subjective perceptions of changes in voice quality were rated according to overall voice grade on a 4-point scale from 0 (normal) to 3 (severe). The lower the overall grade score, the better the quality of voice.

Statistical analysis was performed using SPSS (version 20, IBM Corp., Armonk, N.Y., USA) and Matlab (R2010b, MathWorks Inc., Natick, Mass., USA). A nonparametric 'permutation test' was performed on PIF values and the perceptual voice score rating compared between the two treatment groups. One-tailed tests had significance set at $\mathrm{p} \leq 0.05$. Good interrater agreement was shown for 3 blinded reviewers on overall voice grade ratings (Fleiss's $\kappa=0.53$ ). Assessment on test-retest reliability showed good intrarater consistency (Cronbach's $\alpha=0.71$ ).

\section{Results}

A total of 17 patients were included. There were 5 pacing patients, ranging in age from 41 to 77 years with a mean age of $63.4(\mathrm{SD}=14.4)$. Twelve cordotomy patients were included, ranging in age from 44 to 72 years, with a mean age of 57.6 (SD =10.0). The majority of patients $(94 \%)$ were females. No significant difference was found between the two groups in age or gender. Overall results are detailed in tables 1 and 2. 
Table 1. Outcomes of BVFP patients treated by unilateral laryngeal pacing

\begin{tabular}{lllllll}
\hline & Patient 1 & Patient 2 & Patient 3 & Patient 4 & Patient 5 & Mean \pm SD \\
\hline $\begin{array}{llllll}\text { Sex } \\
\text { Age, years }\end{array}$ & F & F & F & F & F & \\
$\begin{array}{l}\text { PIF, l/s } \\
\quad \text { Preop. }\end{array}$ & 0.00 & 0.60 & 0.70 & 1.39 & 0.00 & $0.54 \pm 0.58$ \\
$\quad \begin{array}{l}\text { Postop. } \\
\text { Grade }\end{array}$ & 1.81 & 0.92 & 1.70 & 1.71 & 1.85 & $1.60 \pm 0.38$ \\
$\quad$ Preop. & 2.00 & 1.00 & 2.00 & 0.00 & 1.00 & $1.20 \pm 0.84$ \\
$\quad$ Postop. & 1.00 & 1.00 & 2.00 & 1.00 & 2.00 & $1.40 \pm 0.55$ \\
\hline
\end{tabular}

Grade = A number from 0 (normal) to 3 (severe) to describe overall voice quality.

Table 2. Outcomes of BVFP patients treated by cordotomy

\begin{tabular}{|c|c|c|c|c|c|c|c|c|c|c|c|c|c|}
\hline & \multicolumn{12}{|c|}{ Patient No. } & \multirow[t]{2}{*}{ Mean \pm SD } \\
\hline & 1 & 2 & 3 & 4 & 5 & 6 & 7 & 8 & 9 & 10 & 11 & 12 & \\
\hline Sex & $\mathrm{F}$ & $\mathrm{F}$ & $\mathrm{F}$ & $\mathrm{F}$ & $\mathrm{F}$ & $\mathrm{F}$ & $\mathrm{F}$ & $\mathrm{F}$ & $\mathrm{F}$ & $\mathrm{F}$ & $\mathrm{F}$ & M & \\
\hline Age, years & 45 & 72 & 53 & 52 & 61 & 65 & 73 & 44 & 62 & 48 & 52 & 64 & $57.6 \pm 10.0$ \\
\hline Postop. PIF, l/s & 0.94 & 0.71 & 0.53 & 0.65 & 1.34 & 1.78 & 1.25 & 1.33 & 1.68 & 0.82 & 1.98 & 1.25 & $1.19 \pm 0.47$ \\
\hline Postop. grade & 2.00 & 2.67 & 3.00 & 3.00 & 3.00 & 1.00 & 1.00 & 1.67 & 3.00 & 2.00 & 1.67 & 3.00 & $2.25 \pm 0.79$ \\
\hline
\end{tabular}

The mean postoperative PIF for pacing patients was $1.60 \mathrm{l} / \mathrm{s}$ (SD = 0.38), which was significantly higher $(\mathrm{p}=0.05)$ than the mean postoperative PIF for cordotomy patients (mean $=1.19$ $\mathrm{l} / \mathrm{s}, \mathrm{SD}=0.47$ ). Preoperative PIF information was available for the 5 pacing patients. Comparison between preoperative and postoperative PIF values in pacing patients showed significant improvement in PIF ( $0.54 \pm 0.58$ vs. $1.60 \pm 0.38, p=0.04)$ as previously reported [26]

Postoperatively, the mean outcome grade score from 0 to 3 for the pacing group was 1.40 $(\mathrm{SD}=0.55)$, which was significantly lower $(\mathrm{p}=0.03)$ than the mean grade score for the cordotomy group $(2.25, \mathrm{SD}=0.79)$. In essence, the overall voice quality outcome was notably better in the pacing patients than in the cordotomy patients. Preoperative grade information was available for the pacing patients. Comparison between preoperative and postoperative grade values in pacing patients showed no significant change $(\mathrm{p}=0.60)$ as previously reported [28].

\section{Discussion}

The aim of this study was to retrospectively compare measurements of pulmonary function and voice quality between patients with bilateral vocal fold paralysis treated with unilateral vocal fold pacing and the current approach of cordotomy. Our results indicate unilateral pacing is better than cordotomy regarding ventilation postoperatively. We selected to use PIF as our main correlate of ventilation, since it is a good index of the mean inspiratory airflow and rate of gas exchange in the lung. During inspiration, negative intrapulmonary pressures create suction forces on the glottis, and increase the resistance to airflow due to the Bernoulli effect and turbulence. Thus, inspiratory flow, rather than expiratory flow, is the 
rate-limiting step in determining the ventilation of the lung [26]. In turn, the magnitude of PIF governs the level of metabolic expenditure and gives an index of the patient activity that can be tolerated. Comparison of postoperative PIF between the two groups revealed significantly higher PIF values in the unilateral pacing group compared to cordotomy patients. While we recognize many factors affect airflow dynamics in a surgical airway [29], our presented results are reasonable given that in the pacing procedure, full cord abduction is restored to one half of the patient's glottis. This varies from the cordotomy procedure in which airflow is minimally increased by creating a graduated opening in a vocal fold.

For the purposes of our retrospective study, PIF was selected as a consistent, objective measure of ventilation. A future prospective investigation should include measurement of additional components of spirometry such as forced expiratory volume at $1 \mathrm{~s}\left(\mathrm{FEV}_{1}\right)$, forced vital capacity (FVC) and the $\mathrm{FEV}_{1} / \mathrm{FVC}$ ratio. Additionally, future analysis would benefit from inclusion of decannulation rates, exercise tolerance quantification, quality of life tools such as a patient-reported dyspnea scale, and patient satisfaction ratings [14, 30-32].

Our results also show a better voice outcome after pacing over cordotomy. Ratings of preand postoperative voice quality were unchanged in the unilateral pacing group. This was to be expected, since the integrity of the vibrating vocal fold is left intact with pacing intervention, and thus voice is preserved. Unfortunately, due to the retrospective design of our study, postintervention data points were not standardized, yet research has shown that voice outcomes after cordotomy can improve over time, with some results not appreciable until 6 or more months after surgery [30]. Therefore, prospective studies warrant standardized postoperative time points for finer analy-sis. Additionally, while performed blinded in this study, overall voice grade is a subjective measure of voice now considered somewhat 'outdated' compared to the newer rating system Consensus Auditory-Perceptual Evaluation of Voice [33]. Therefore, this and more objective measures, such as harmonic-to-noise ratio, maximum phonation time, and maximum and minimum vocal intensities, could be considered for future studies.

These findings represent the first published comparison between unilateral laryngeal pacing and cordotomy for patients with BVFP. Our study demonstrated unilateral pacing to be an effective means of treatment, superior to cordotomy with respect to outcome measures of ventilation and voice.

\section{Acknowledgment}

David L. Zealear has received NIH grants R01-DC001149 and R01-DC008429 to support this research.

\section{Disclosure Statement}

No conflicts of interest.

\section{References}

- 1 Benninger MS, Gillen JB, Altman JS: Changing etiology of vocal fold immobility. Laryngoscope 1998;108:13461350.

- 2 Dedo H: The paralyzed larynx: an electromyographic study in dogs and humans. Laryngoscope 1970;80: 1455-1517.

3 Holinger L, Holinger PC, Holinger PH: Etiology of the bilateral abductor vocal cord paralysis. Ann Otol Rhinol Laryngol 1976;85:428-436.

4 Sapundzhiev N, Lichtenberger G, Eckel HE, Friedrich G, Zenev I, Toohill RJ, Werner JA: Surgery of adult bilateral vocal fold paralysis in adduction: history and trends. Eur Arch Otorhinolaryngol 2008;265:1501-1514. 
5 Viau F, Ledente A, Le Tinier JY: Complications of tracheotomy. Rev Pneumol Clin 1988;44:24-32.

- 6 Goldenberg D, Ari EG, Golz A, Danino J, Netzer A, Joachims HZ: Tracheotomy complications: a retrospective study of 1,130 cases. Otolaryngol Head Neck Surg 2000;123:495-500.

- 7 Norwood S, Vallina VL, Short K, Saigusa M, Fernandez LG, McLarty JW: Incidence of tracheal stenosis and other late complications after percutaneous tracheostomy. Ann Surg 2000;232:233-241.

- 8 Lichtenberger G, Toohill RJ: Technique of endo-extralaryngeal suture lateralization for bilateral abductor vocal cord paralysis. Laryngoscope 1997;107:1281-1283.

- 9 Andrade Filho PA, Rosen CA: Bilateral vocal fold paralysis: an unusual treatment with botulinum toxin. J Voice 2004;18:254-255.

10 Ossoff RH, Sisson GA, Moselle HI, et al: Endoscopic laser arytenoidectomy for the treatment of bilateral vocal cord paralysis. Laryngoscope 1984;94:1293-1297.

11 Crumley RL: Endoscopic laser medial arytenoidectomy for airway management in bilateral laryngeal paralysis. Ann Otol Rhinol Laryngol 1993;102:81-84.

12 Woodson G, Weiss T: Arytenoid abduction for dynamic rehabilitation of bilateral laryngeal paralysis. Ann Otol Rhinol Laryngol 2007;116:483-490.

13 Woodson G: Arytenoid abduction: indications and limitations. Ann Otol Rhinol Laryngol 2010;119:742-748.

14 Dennis DP, Kashima H: Carbon dioxide laser posterior cordectomy for treatment of BVCP. Ann Otol Rhinol Laryngol 1989;98:930-934.

15 Paniello RC: Laryngeal reinnervation. Otolaryngol Clin North Am 2004;37:161-181.

16 Marie J, Lacoume Y, Magnier P, et al: Selective bilateral motor reinnervation of the canine larynx. Laryngorhinootologie 2000;79:S188-S189.

17 Marina MB, Marie JP, Birchall MA: Laryngeal reinnervation for bilateral vocal fold paralysis. Curr Opin Otolaryngol Head Neck Surg 2011:19:434-438.

18 Marie JP: Nerve reconstruction; in Remacle M, Eckle HE (eds): Surgery of Larynx and Trachea. Heidelberg, Springer, 2010, pp 279-294.

19 Marie JP, Dehesdin D, Ducastelle T, Senant J: Selective reinnervation of the abductor and adductor muscles of the canine larynx after recurrent nerve paralysis. Ann Otol Rhinol Laryngol 1989;98:530-536.

20 Kwak PE, Friedman AD, Lamarre ED, Lorenz RR: Selective reinnervation of the posterior cricoarytenoid and interarytenoid muscles: an anatomical study. Laryngoscope 2010;120:463-467.

21 Pearce EC, Mainthia R, Garrett CG, Ossoff RH, Francis DO: Current management and treatment of bilateral vocal fold paralysis. In preparation.

22 Maurizi M, Paludetti F, Galli J, Cosenza A, Di Girolamo S, Ottaviani F: $\mathrm{CO}_{2}$ laser subtotal arytenoidectomy and posterior true and false cordotomy in the treatment of post-thyroidectomy bilateral laryngeal fixation in adduction. Eur Arch Otorhinolaryngol 1999;256:291-295.

-23 Eckel HE, Thumfart M, Wassermann K, Vossing M, Thumfart WF: Cordectomy versus arytenoidectomy in the management in bilateral vocal cord paralysis. Ann Otol Rhinol Laryngol 1994;103:852-857.

-24 Lawson G, Remacle M, Hamoir M, Jamart J: Posterior cordectomy and subtotal arytenoidectomy for the treatment of bilateral vocal fold immobility: functional results. J Voice 1996;10:314-319.

25 Pia F, Pisani P, Aluffi P: $\mathrm{CO}_{2}$ laser posterior ventriculocordectomy for the treatment of bilateral vocal fold paralysis. Eur Arch Otorhinolaryngol 1999;256:403-406.

-26 Zealear DL, Billante CR, Courey MS, Netterville JL, Paniello RC, Sanders I, Herzon GD, Goding GS, Mann W, Ejnell H, Habets AM, Testerman R, Van de Heyning P: Reanimation of the paralyzed human larynx with an implantable electrical stimulation device. Laryngoscope 2003;113:1149-1156.

27 Hirano M: Clinical Examination of Voice. New York, Springer, 1981.

28 Billante CR, Zealear DL, Courey MS, Netterville JL: Effect of chronic electrical stimulation of laryngeal muscle on voice. Ann Otol Rhinol Laryngol 2002;111:328-332.

29 Gökcan MK, Kurtuluş DF, Ustüner E, Ozyürek E, Kesici GG, Erdem SC, Dursun G, Yağci C: A computational study on the characteristics of airflow in bilateral abductor vocal fold immobility. Laryngoscope 2010;120:18081018.

-30 Hans S, Grevier-Buchanan L, Laccourreye O, Brasnu D: Aerodynamic and acoustic parameters in $\mathrm{CO}_{2}$ laser posterior transverse cordotomy for bilateral vocal fold paralysis. Acta Otolaryngol 2000;120:330-335.

31 Dursen G, Gokcan K: Aerodynamic, acoustic and functional results of posterior transverse laser cordotomy for bilateral abductor vocal cord paralysis. J Laryngol Otol 2006;120:282-288.

-32 Harnisch W, Brosch S, Schmidt M, Hagen R: Breathing and voice quality after surgical treatment for bilateral vocal cord paralysis. Arch Otolaryngol Head Neck Surg 2008;134:278-284.

33 Zraick RI, Kempster GB, Connor NP, Thibeault S, Klaben BK, Bursac Z, Thrush CR, Glaze LE: Establishing validity of the Consensus Auditory-Perceptual Evaluation of Voice (CAPE-V). Am J Speech Lang Pathol 2011; 20:14-22. 